Hanna Kwiendacz ${ }^{1} \mathbb{D}$, Katarzyna Nabrdalik ${ }^{1} \mathbb{D}$, Zenon Brzoza ${ }^{\mathbb{D}}$, Iga Stokłosa ${ }^{3}$,

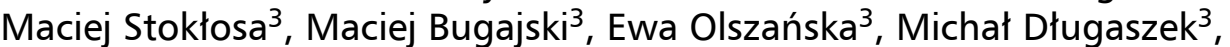
Karolina Drożdż ${ }^{1}$ D, Weronika Hajzler ${ }^{3}$, Bożena Rabowicz ${ }^{1}$, Janusz Gumprecht ${ }^{1}$ (D)

${ }^{1}$ Department of Internal Medicine, Diabetology and Nephrology, Faculty of Medical Sciences in Zabrze, Medical University of Silesia, Katowice, Poland ${ }^{2}$ Department of Internal Diseases and Allergology, Institute of Medicine, University of Opole, Opole, Poland

${ }^{3}$ Students' Scientific Association by the Department of Internal Medicine, Diabetology and Nephrology, Faculty of Medical Sciences in Zabrze, Medical University of Silesia, Katowice, Poland

\title{
Knowledge about diabetes mellitus among Polish medical students
}

\section{ABSTRACT}

Background. Due to high prevalence of diabetes mellitus all over the world it is essential for students who will become doctors of different specialties to possess a basic knowledge of it in this field. This pilot surveybased study was designed to assess diabetes related knowledge among faculty of medicine students from all of the medical universities in Poland.

Materials and methods. Students were invited to fulfill the questionnaire during Students' Diabetology Conference and via the Internet (social media). The survey consisted of questions about respondent's age and personal history of diabetes and diabetes related knowledge (etiology, symptoms, risk factors, complications and treatment of diabetes mellitus and additionally a section concerning gestational diabetes). Results. A total number of $\mathbf{1 2 0 0}$ medical students from Poland (70\% women; mean age [SD] 22.12 [1.83] years of age) completed the survey. Mean test result was $66.62 \%$. The best score was observed in the group of students enrolled in the $5^{\text {th }}$ and $6^{\text {th }}$ year of study, and those with diabetes mellitus type 1 .

Address for correspondence:

lek. Hanna Kwiendacz

Katedra i Klinika Chorób Wewnętrznych,

Diabetologii i Nefrologii

Wydział Nauk Medycznych w Zabrzu

Śląski Uniwersytet Medyczny w Katowicach

ul. 3 Maja 13-15, 41-800 Zabrze

Phone: +48 323704 488, fax: +48 323704489

e-mail: hanna.kwiendacz@gmail.com

Clinical Diabetology 2020, 9, 4, 245-252

DOI: $10.5603 /$ DK.2020.0023

Received: 07.03.2020

Accepted: 05.04.2020
Conclusions. The study outcome proves that knowledge about diabetes mellitus among surveyed medical students in Poland is insufficient, therefore persistent improvement in transmitting it during the course of medical education is essential. (Clin Diabetol 2020; 9; 4: 245-252)

Key words: knowledge, diabetes mellitus, medical students, education, survey

\section{Introduction}

Among the most challenging health problems of the XXI century diabetes mellitus is one of the leaders [1]. It is estimated that 425 million adults all over the world are suffering from diabetes, whereas every second patient is undiagnosed [1]. According to the International Diabetes Federation (IDF), 629 million people in the world will have diabetes until the year 2045 [1]. In the Polish society, approximately 2.6 million people (i.e. almost $7 \%$ of the Polish population) are suffering from diabetes and $40 \%$ of patients are still undiagnosed [2]. The disease, especially when poorly controlled, may lead to many micro- and macrovascular complications. Diabetes doubles the risk of a cardiovascular disease and patients are prone to microvascular complications such as retinopathy, nephropathy and neuropathy what implicates the necessity for a patient to be treated by doctors of many different specialties. It is important to notice that diseases of different organs, (e.g. coronary artery disease, stroke, urogenital infections, foot ulcer) may be the first symptom of undiagnosed diabetes and doctors should advice the patient to have the blood glucose concentration tested [3]. 
In the light of constantly increasing number of patients suffering from diabetes who visit many different health specialist during their lifespan, it is essential to propagate the broadest knowledge of the risk factors, symptoms and complications as well as treatment methods of the disease among medical students [1, 4, 5]. Being up to date with the guidelines and an ability to use obtained knowledge in practice is essential to ensure proper diabetes care [2]. An appropriate attention should be paid particularly to education in the field of risk factors and symptoms of diabetes mellitus in order to diagnose the disease early enough to prevent or delay its complications [6, 7]. During the past 10 years, there were only several studies performed in countries like Pakistan, India, Libya, Nepal, Saudi Arabia, Nigeria, Switzerland, Great Britain, Germany, United Stated of America and Poland which investigated medical students' knowledge related to diabetes [4-12]. These surveys included from 60 to approximately 400 respondents but to our knowledge, neither one evaluated diabetes related knowledge among students from the first to the last year of medical training, coming from all of the academic centers in one country.

The 6-year course of medical education in Poland is divided into a "preclinical" period (year $1^{\text {th }}$ and $2^{\text {nd }}$ ) and a "clinical" one (years $3^{\text {rd }}-6^{\text {th }}$ ). Knowledge about pathophysiology of the disease is obtained during a preclinical phase, whereas information about treatment, complications and prognosis is gained in further years of education. Knowledge gained during medical studies is crucial for young doctors and in some medical specialties university education is the only time in once career to learn about diabetes mellitus [12]. The aim of this study was to assess diabetes related knowledge among faculty of medicine students from all of the medical universities in Poland.

\section{Material and methods}

This was a survey based, multi-centered study conducted among Polish medical faculty students from all medical universities in Poland during the Diabetology Conference in Zabrze on $21^{\text {st }}$ of November 2015 organized by the International Medical Federation of Students' Association IFMSA-Poland. After the Conference ended the survey assessing diabetes related knowledge was shared among faculty of medicine students studying in all of medical universities in Poland by social media as this form was the best possible way to reach respondents from all of the country. Each medical university representative student attending the Conference was asked to carry on the survey to their fellow students in the representative's university throughout the Internet with the use of Google Forms till the $6^{\text {th }}$ of December 2015. The questionnaire was self-prepared and approved by two independent experts in diabetology field and was pre-tested on a group of 60 randomly selected students of the Medical University of Silesia in Katowice. Underlying components were identified using principal component analysis and the internal consistency of questions was checked. The questionnaire consisted of 19 questions, including 13 single-choice questions and 6 multiple-choice questions and was divided into the following categories: etiology, symptoms, risk factors, complications and treatment of diabetes mellitus and a section concerning gestational diabetes (Table 1). Evaluation process of the survey was performed in the following manner: a single-choice question was awarded 1 point for a correct answer, 0 points for a wrong one or the answer "I do not know"; each multiple-choice question was awarded 1 point for each correctly selected answer and minus 1 point for a wrong answer indicated. One could score up to 33 points in the pool. Students were also asked to indicate their age, a personal history of diabetes mellitus and the year of studies. Participants were split into two groups depending on the year of study ("preclinical group" which is $1^{\text {st }}-2^{\text {nd }}$ year of facuIty of medicine and "clinical group" which is $3^{\text {rd }}-6^{\text {th }}$ year of faculty of medicine).

The results obtained were analyzed using STATISTICA 12.5 (StatSoft, Cracow, Poland). Quantitative variables were compared using Mann-Whitney $U$ test. Kruskal-Wallis test was used to compare the multiple groups and the Spearman's rank correlation to verify the relationship between variables. A P value of $<0.05$ was considered significant.

\section{Results}

1200 (70\% women; mean age [SD] 22.12 [1.83] years of age) students from all of the medical universities in Poland completed our diabetic knowledge test, which is approximately several percent of all the medical students in Poland. $1.7 \%(n=20)$ of the respondents declared to have type 1 diabetes mellitus and none declared to have type 2 of the disease. Mean result obtained from the test was $66.62 \%$, whilst the worst one was $29.4 \%$ and the best was $100 \%$. The distribution of the results in the study cohort are presented according to the year of study (Figure 1). There was no significant difference in the test result in relation to gender $(66.53 \pm 11.6 \%$ vs. $66.64 \pm 11.12 \%$; $\mathrm{P}>0.05)$ in men and women respectively. The higher was the student's year of study, the better was the test result score (Figure 1). Students who started or continued clinical subjects achieved significantly higher result in comparison with "preclinical" students' group 
Table 1. Diabetes related knowledge questionnaire (correct answers are in italics)

1. Is diabetes mellitus (DM) an infectious disease?
a) Yes
b) No
c) I do not know

2. Insulin:
a) Increases blood glucose concentration
b) Decreases blood glucose concentration
c) Does not affect the blood glucose concentration
d) I do not know how it affects blood glucose concentration
e) I do not know what insulin is

3. Too low blood glucose concentration:
a) It does not affect one's health
b) May be dangerous for life
c) Causes cancer
d) It is beneficial in DM because it makes it easier to lose unnecessary kilograms
e) I do not know

4. What is a proper fasting plasma glucose concentration?
a) Less than $70 \mathrm{mg} / \mathrm{dl}$
b) $70-99 \mathrm{mg} / \mathrm{dl}$
c) $100-125 \mathrm{mg} / \mathrm{dl}$
d) Above $125 \mathrm{mg} / \mathrm{dl}$
e) I do not know

5. What are the risk factors of DM type 2? More than 1 answer is correct:
a) Inadequate diet
b) Smoking cigarettes
c) Sedentary lifestyle
d) Alcohol consumption
e) Hypertension
f) Genetic predisposition
g) Obesity
h) Frequent use of antibiotics
i) I do not know

6. What may be the symptoms of DM? More than 1 answer is correct:
a) Polyuria
b) Oliguria
c) Polydipsia
d) Oligodipsia
e) Increased activity
f) Somnolence
g) Increased appetite
h) Blurred vision
i) Muscle pain
j) Unexpected weight loss
k) I do not know

7. What can untreated or poorly controlled diabetes lead to? More than 1 answer is correct:
a) Diabetic nephropathy
b) Sepsis
c) Coronary artery disease
d) Vascular damage
e) Osteoporosis
f) Alzheimer's disease 
Table 1 (cont.). Diabetes related knowledge questionnaire (correct answers are in italics)
g) Blindness
h) Stomach cancer
i) Diabetic foot
j) I do not know

8. Is it possible to die from complications of DM?
a) Yes
b) No
c) Yes, but very rarely
d) I do not know

9. Is correct blood glucose concentration important for pregnant women?
a) Yes
b) No
c) I do not know

10. Should pregnant women be screened for DM?
a) Yes
b) No
c) I do not know

11. What is the basic treatment of DM besides medications?
a) Medications only
b) Physical effort
c) Proper diet
d) $B$ and $C$
e) I do not know

12. What can be used to treat DM?
a) Insulin
b) Oral medications
c) Diet
d) All listed
e) I do not know

13. What is the first choice treatment in DM type 1 ?
a) Metformin
b) Sulphonylurea
c) Insulin
d) Acarbose
e) DPP-4 inhibitors
f) I do not know

14. Apart from dietary intervention, how can gestational diabetes mellitus (GDM) be treated?
a) Sulphonylurea
b) Acarbose
c) Gliflozins
d) Insulin
e) GLP-1 receptor agonists
f) I do not know

15. Glycated hemoglobin $\left(\mathrm{HbA}_{1 \mathrm{c}}\right)$ determines the average blood glucose concentration during past:
a) Week
b) 2 weeks
c) 1 month
d) 3 months
e) Half a year
f) I do not know 
Table 1 (cont.). Diabetes related knowledge questionnaire (correct answers are in italics)

16. The criterion for good controlled DM type 2 for general population is the $\mathrm{HbA}_{1 c}$ level:
a) $\leq 6.0 \%$
b) $\leq 6.5 \%$
c) $\leq 7 \%$
d) $\leq 8.0 \%$
e) $\leq 10.0 \%$
f) I do not know

17. How can insulin be administered? More than 1 answer is correct
a) Intradermally
b) Subcutaneously
c) Intramuscularly
d) Intravenously
e) Orally
f) I do not know

18. Which insulin could be administrated intravenously? More than 1 answer is correct
a) Lispro
b) Neutral insulin
c) NPH
d) Glargine
e) Insulin cannot be administered intravenously
f) I do not know

19. What are the differences between human and analogue insulin?

a) Human insulin can be injected just before a meal, analog insulin should be injected 30 minutes before a meal

b) Human insulin is injected just like an analogue insulin after the meal

c) Human insulin should be injected half an hour before a meal and analogue insulin can be injected just before a meal

d) There is no need to eat snacks with human insulin

e) I do not know

$\mathrm{DM}$ - diabetes mellitus; GDM - gestational diabetes mellitus; $\mathrm{HbA}_{1 c}$ - glycated hemoglobin

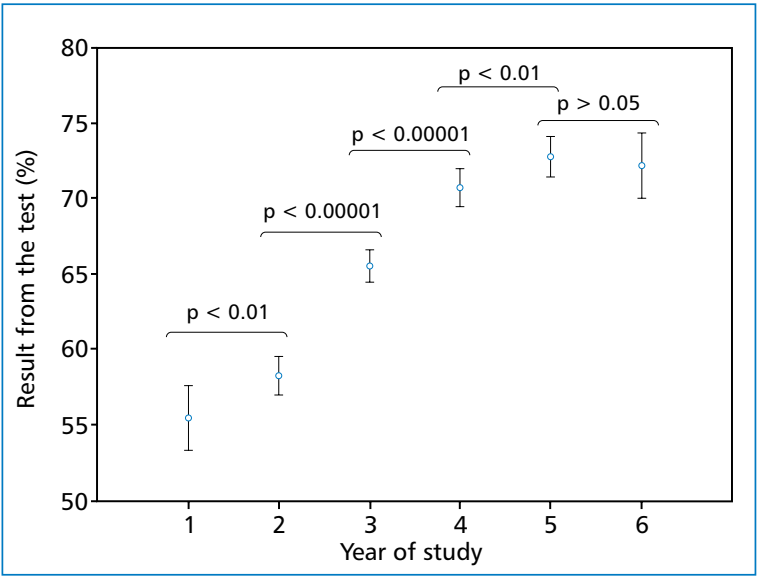

Figure 1. The distribution of the results in the study cohort according to the year of study

$(70 \pm 9.85$ vs. $57.56 \pm 9.59 ; \mathrm{P}=0.0000)$ (Table 2$)$. As a conclusion, being in the group of clinical education, higher year of study and having type 1 diabetes mellitus
$(P=0.0084)$ were the factors significantly associated with better results of the diabetes knowledge test.

\section{Discussion}

According to our best knowledge, this investigation is the biggest multi-centered study not only in Poland, but also worldwide, since we have obtained 1200 respondents studying medicine faculty from the $1^{\text {st }}$ to the $6^{\text {th }}$ year of study from all of the medical universities in Poland. As patients suffering from diabetes mellitus are prone to many complications which are treated by doctors of different specialties, the basic knowledge of diabetes mellitus is crucial for every doctor. It might happen that before a patient visits a diabetologist, they are first seen by a general physician, surgeon, urologists, ophthalmologist, cardiologist, nephrologist or neurologist who should be able to diagnose the disease properly based on declared symptoms. Taking into consideration that the prevalence of diabetes is rising, medical students should be well-educated about the disease during their course of studies. 
Table 2. Percentage of students answering correctly about risk factors, symptoms and complications of diabetes with regard to preclinical and clinical level of education

\begin{tabular}{lccc}
\hline Variable & Preclinical students & Clinical students & P value \\
\hline Risk factors & & & \\
Unhealthy diet & $96 \%(n=294)$ & $96 \%(n=792)$ & 0.9522 \\
Sedentary lifestyle & $89 \%(n=273)$ & $95 \%(n=777)$ & 0.0040 \\
Hypertension & $32 \%(n=97)$ & $36 \%(n=296)$ & 0.1897 \\
Genetic predisposition & $76 \%(n=233)$ & $89 \%(n=731)$ & 0.0000 \\
Obesity & $95 \%(n=292)$ & $99 \%(n=815)$ & 0.0005 \\
Symptoms of diabetes & & & 0.0000 \\
Polyuria & $74 \%(n=229)$ & $97 \%(n=793)$ & 0.0000 \\
Polydipsia & $89 \%(n=273)$ & $99 \%(n=812)$ & 0.0369 \\
Somnolence & $91 \%(n=277)$ & $94 \%(n=776)$ & 0.0000 \\
Blurred vision & $56 \%(n=174)$ & $73 \%(n=600)$ & 0.0000 \\
Unexpected weight loss & $41 \%(n=125)$ & $63 \%(n=518)$ & \\
Complications of diabetes & & & 0.0000 \\
Diabetic nephropathy & $87 \%(n=265)$ & $98 \%(n=799)$ & 0.0000 \\
Coronary artery disease & $54 \%(n=166)$ & $69 \%(n=566)$ & 0.0000 \\
Vascular damage & $73 \%(n=224)$ & $96 \%(n=784)$ & 0.0000 \\
Blindness & $85 \%(n=260)$ & $98 \%(n=801)$ & 0.0444 \\
Diabetic foot & $97 \%(n=297)$ & $99 \%(n=815)$ & \\
\hline
\end{tabular}

$\mathrm{n}-$ number. A P value of $<0.05$ is considered statistically significant

Studies related to medicine faculty students' knowledge about diabetes performed up to date are scarce and it is impossible to directly compare the outcomes because of different groups of respondents and different surveys applied. For this reason we will only refer to them to present just a general insight into studies dealing with assessment of medical faculty students' awareness of diabetes. The general level of diabetes related knowledge assessed in the presented research is unsatisfying. The lowest score was achieved by a $1^{\text {st }}$ year medical students, which reflects the fact that people entering universities have very little knowledge of diabetes. Only few students achieved $100 \%$ score, and one might suspect that it could be due to an individual interest in diabetology or having to deal on everyday basis with a relative having diabetes. Students have essential problems with marking correctly all risk factors, symptoms and type of insulin applied intravenously. What is worth emphasizing, only $50 \%$ of respondents knew what medications could be administered to pregnant woman suffering from diabetes. Sagar et al., in a study conducted in Libya testing the knowledge of 325 final year medical students using an Arabic 24-item "Short Diabetes Knowledge Test", assessed students' knowledge as reasonable (mean score $76.7 \%$ ), with major deficiencies in dietary management [8]. Our respondents demonstrated knowledge on a lower average level (mean score 66.6\%), however, it is impossible to compare them directly as the surveys differed. Yet the general author's conclusion was that students in Libya have a proper working knowledge of diabetes and would be able to care for patients [8]. An opposite observation was made by Lansang et al. from the University of Florida in United States of America, who noticed that medical students are unable to translate theoretical knowledge into practice [13]. Students in that study were able to diagnose diabetes but they could not indicate appropriate scheme of treatment. Students who took part in our research recognized symptoms of hyperglycemia properly, however our respondents had significant problems with questions related to insulin use, which was also observed in the American research indicated above. In the context of a high number of people with hypertension, medical student should be aware that it is a risk factor for diabetes [14], the more so that hypertension was poorly recognized as a risk factor among entire study group of our students. Students' knowledge should be at least satisfactory regarding symptoms of diabetes mellitus what is essential for patients prompt diagnosis which nowadays is still delayed. Both preclinical and clinical students associated diabetes with somnolence $(91 \%$ of preclinical students vs. $95 \%$ of clinical ones) and polydipsia ( $89 \%$ vs. 99\%). The least known symptom of diabetes in both groups was an unexpected weight loss ( $41 \%$ vs. $63 \%$ ) and visual impairment (56\% vs. $73 \%$ ). 
Our findings are in accordance with other researchers', one of whom is Mumtaz et al., in Pakistan, as the most frequently marked symptoms in their study were also polyuria and polydipsia among preclinical and clinical students ( $81 \%$ vs. $90 \%$ respectively) while weight loss was associated with diabetes by $39 \%$ of preclinical students and by $57 \%$ clinical students, which is also comparable to our results [4]. As a conclusion, in different countries both polyuria and polydipsia are recognized by majority of students as a potential symptom of diabetes, however, sign such as unexpected weight loss is overlooked. In the field of identifying complications, pointing coronary disease as one of them, was problematic for both clinical and preclinical groups of students. Only $68 \%$ of clinical students and $53 \%$ of preclinical students responded correctly to that question $(\mathrm{P}<0.001)$. In the Pakistani study similar results were obtained with $72 \%$ of the clinical and $51 \%$ of the preclinical students being able to indicate the diabetes complications correctly [4]. Among our respondents $1.7 \%$ declared to suffer from type 1 diabetes mellitus and they obtained a better result from the test compared to healthy colleagues ( $73.4 \%$ vs. $66.5 \%)$, which is obviously explained by the fact that people having diabetes are supposed to be educated while treatment. A study conducted in Saudi Arabia showed a significant correlation between gender and the level of knowledge about diabetes, where men have obtained a better result [9]. In our study there was no significant difference between the level of knowledge among men and women. It is worth mentioning that the gap in knowledge related to pointing hypertension as a risk factor of diabetes and weight loss as a symptom of the disease is the same as in general population what we have proven in our previous research [15]. Taking into consideration all students attending medicine course in Poland, our respond rate is estimated to be a couple of percent, which is the main limitation of our study. It is difficult to preciously assess the number of students in medical faculty each year because not every university publishes such data. On the other hand, it is just a pilot study and it already has the biggest total number of medicine faculty students in Poland and worldwide and the poor results obtained enquire to continuation of research in this field. The low response rate is a well-recognized limitation of Web-based survey [16], which gives the possibility to reach many different parts of the country as well as bigger number of people that paper surveys, however not every respondent expresses a desire to take part in this kind of research. Similar observations related to low response rate to invitation related to participation in the questionnaire based study come from our previous research related to diabetes knowledge assessment where we examined Polish mountain guides [17] and people from the general community [15].

\section{Conclusions}

It the light of constantly increasing prevalence of diabetes mellitus which affects many organs, medical faculty students, who will became doctors of different specialties should gain a basic diabetes related knowledge during their university studies. Barring in mind the limitation of the ability to reach the high response rate, the presented study indicates that there are gaps in students' knowledge related to diabetes what needs a future attention and indicates a need for persistent improvement in spreading diabetology knowledge during medical education in order to struggle more efficiently against diabetes epidemic.

\section{Conflict of interest}

The authors declare to have no conflict of interest.

\section{REFERENCES}

1. International Diabetes Federation. IDF Diabetes Atlas Eighth edition 2017. http://fmdiabetes.org/wp-content/uploads/2018/03/ IDF-2017.pdf. Accessed November 14, 2017.

2. Araszkiewicz A, Bandurska-Stankiewicz E, Budzyński A, et al. 2019 Guidelines on the management of diabetic patients. A position of Diabetes Poland. Clinical Diabetology. 2019; 8(1): 1-95, doi: 10.5603/dk.2019.0001.

3. Chen R, Ovbiagele B, Feng W. Diabetes and stroke: epidemiology, pathophysiology, pharmaceuticals and outcomes. Am J Med Sci. 2016; 351(4): 380-386, doi: 10.1016/j.amjms.2016.01.011, indexed in Pubmed: 27079344.

4. Mumtaz S, Ashfaq T, Siddiqui H. Knowledge of medical students regarding diabetes mellitus at Ziauddin University, Karachi. J Pak Med Assoc. 2009; 59: 163-166. https://jpma.org.pk/PdfDownload/1645 Accessed March 2009.

5. Amans RAF, Nazareth F, Arunachalam R et al. Study of awareness about diabetes mellitus among undergraduate medical students. International Journal of Recent Trends in Science and Technology 2014; 12: 491-493. https://pdfs.semanticscholar.org/ b5d9/94f64762cdef389b1ce69ef5c1d1d883154e.pdf Accessed October 14, 2014.

6. Tamunopriye J. The effectiveness of education on awareness and knowledge of childhood diabetes amongst medical students in port harcourt. British Journal of Education, Society \& Behavioural Science. 2015; 5(4): 410-415, doi: 10.9734/bjesbs/2015/14368.

7. Rubin D, Moshang J, Jabbour S. Diabetes knowledge: are resident physicians and nurses adequately prepared to manage diabetes? Endocr Pract. 2007; 13(1): 17-21, doi: 10.4158/ep.13.1.17.

8. Sagar AE. Practical diabetes knowledge of final-year medical students in Tripoli, Libya. Ibnosina Journal of Medicine and Biomedical Sciences. 2011; 3(1): 36-41, doi: 10.4103/1947-489x.210850.

9. Al Wadaani FA. The knowledge attitude and practice regarding diabetes and diabetic retinopathy among the final year medical students of King Faisal University Medical College of Al Hasa region of Saudi Arabia: a cross sectional survey. Niger J Clin Pract. 2013; 16(2): 164-168, doi: 10.4103/1119-3077.110133, indexed in Pubmed: 23563455.

10. Hessett C, Moran A, Boulton AJ. An evaluation of diabetes knowledge amongst general practitioners and senior medical students. Central Manchester Health Authority Working Party on Diabetes Care. Diabet Med. 1989; 6(4): 351-353, doi: 10.1111/j.14645491.1989.tb01179.x, indexed in Pubmed: 2524343. 
11. Holstein A, Widjaja A, Nahrwold D, et al. Clinically relevant diabetes knowledge and experience amongst final year medical students. Practical Diabetes International. 2000; 17(1): 3-8, doi: 10.1002/(sici)1528-252x(200001)17:1<3::aid-pdi15>3.0.co;2-5.

12. Piłaciński S, Czyż K, Malida J, et al. Diabetes related knowledge assessment among 5th and 6 th year medical students. Nowiny Lekarskie. 2003; 72: 37-41.

13. Lansang $M C$, Harrell $H$. Knowledge on inpatient diabetes among fourth-year medical students 2005-2006. Diabetes Care. 2007; 30(5): 1088-1091, doi: 10.2337/dc06-2174, indexed in Pubmed: 17325263.

14. Kim MJ, Lim NK, Choi SJ, et al. Hypertension is an independent risk factor for type 2 diabetes: the Korean genome and epidemiology study. Hypertens Res. 2015; 38(11): 783-789, doi: 10.1038/hr.2015.72.
15. Nabrdalik K, Kwiendacz H, Florczyk I, et al. Knowledge about type 2 diabetes among visitors of two shopping centers in Upper Silesia, Poland: a survey research performed as a part of social action "Health under Control". Clinical Diabetology. 2018; 7(4): 182-188, doi: 10.5603/dk.2018.0017.

16. Aitken $C$, Power $R$, Dwyer R. A very low response rate in an on-line survey of medical practitioners. Aust N Z J Public Health. 2008; 32(3): 288-289, doi: 10.1111/j.1753-6405.2008.00232.x, indexed in Pubmed: 18578832.

17. Nabrdalik K, Kwiendacz H, Gubała M, et al. Diabetes-Related knowledge of polish national mountain leaders. High Alt Med Biol. 2018; 19(3): 237-243, doi: 10.1089/ham.2017.0168, indexed in Pubmed: 29924643. 